\title{
Synthesis of Amorphous Polylactide and Poly (lactide-co-glycolide) Containing High L-form Enantiomer for Use in Controlled Release Drug Delivery
}

\author{
NUANCHAI KHOTSAENG, KANSIRI PAKKETHATI and YODTHONG BAIMARK*
}

\author{
Biodegradable Polymers Research Unit, \\ Department of Chemistry and Center of Excellence for Innovation in Chemistry, \\ Faculty of Science, Mahasarakham University, Mahasarakham 44150, Thailand. \\ ${ }^{*}$ Corresponding author E-mail: yodthong.b @ msu.ac.th
}

http://dx.doi.org/10.13005/ojc/320503

(Received: September 08, 2016; Accepted: October 12, 2016)

\begin{abstract}
In this work, the effect of L-lactide (LL) copolymerization on the properties of poly(D,L-lactide) (PDLL) and poly(D,L-lactide-co-glycolide) (PDLLG) copolymers and its drug release behaviors were determined and discussed. The copolymers were synthesized by ring-opening polymerization of DLL, LL, and G monomer mixtures. The PDLL with DLL/LL ratios of 100/0-50/50 by mole and the PDLLG with DLL/LL/G ratios of 75/0/25-37.5/37.5/25 by mole were investigated. All the copolymers were completely amorphous. The drug-loaded copolymer microparticles with a spherical shape and smooth surface were prepared by the oil-in-water emulsion solvent evaporation method. Indomethacin was used as a poorly-water soluble model drug. The copolymerization of the LL monomer did not change the in vitro drug release profiles of the PDLL and the PDLLG microparticles significantly. It is suggested that these amorphous PDLL and PDLLG copolymers that contain higher L enantiomer amounts have the potential to be developed further as a lower-cost PDLL and PDLLG, respectively, for use as controlled release drug delivery systems.
\end{abstract}

Keywords: Lactide, Glycolide, Copolymers, Microparticles, Drug delivery.

\section{INTRODUCTION}

Controlled release drug delivery systems made from biodegradable particles provide several benefits over traditional formulations. ${ }^{1}$ Prior to release, the drug is protected from degradation or premature metabolism by the polymeric particle matrix. The release of the drug is sustained over days to months, thereby keeping the drug concentration in the plasma at an effective level for longer periods of therapy time and reducing the toxic side-effects from overdose of the drug. This decreases the frequency of administration and increases patient compliance. ${ }^{2}$ Biodegradable microspheres for drug delivery have been widely made from a variety of biodegradable polyesters due to their biodegradability and biocompatibility. The removal of these biodegradable polyester-based microspheres at the end of the 
therapy is not required. Administration of medication via such a system is advantageous because the microspheres can be ingested or injected ${ }^{3-4}$

Amorphous poly(D,L-lactide) (PDLL) and poly(D,L-lactide-co-glycolide) (PDLLG) are biodegradable and biocompatible polyesters that have been widely investigated for use as controlled release drug delivery systems. ${ }^{4-6}$ This is due to the fact that good drug distribution into the amorphous PDLL and PDLLG matrices can be obtained. The semi-crystalline phases in the poly(L-lactide) (PLL) matrix may induce drug aggregation. A good distribution of the entrapped drug into the microparticle matrices could allow a consistent drug release rate. Controllable molecular weights of the PDLL and the PDLLG are usually synthesized by ring-opening polymerization of DLL and DLL/G monomers, respectively. The higher hydrophilic $G$ units in the PDLLG resulted in a faster biodegradation rate than the PDLL.

An equivalent $\mathrm{D}$-lactic acid/L-lactic acid mixture, called D,L-lactic acid, is used to prepare the DLL monomer. However, L-lactic acid, the monomer precursor of LL, is produced at a larger scale for supply to food, cosmetic, and pharmaceutical applications. Therefore, the L-lactic acid is easier to find and cheaper than the D,L-lactic acid. The DLL and LL monomers are prepared from the D,Llactic acid and the L-lactic acid, respectively, by the same procedure. This has the benefit of offering a substantial reduction in cost by the addition of LL to produce lower-cost alternatives to the current commercial PDLL and PDLLG for use in controlled release drug delivery applications.

In the present paper, PDLL and PDLLG copolymers with different LL monomer contents were synthesized by ring-opening polymerization of the DLL/LL and the DLL/LL/G mixtures, respectively. We prepared drug-loaded copolymer microparticles using an oil-in-water emulsion solvent evaporation method. Indomethacin was used as the hydrophobic model drug. The characteristics of the drug-loaded PDLL and PDLLG microparticles containing high LL content and in vitro indomethacin release were determined and compared to the neat PDLL and PDLLG microparticles.

\section{Experimental section Materials}

Crude D,L-lactide (DLL) and L-lactide (LL) monomers were synthesized from D,L-lactic acid (85\% w/v, 50/50 D-/L-form ratio, Acros Organics) and L-lactic acid (88\% w/v, 5/95 D-/L-form ratio, Purac), respectively, by direct polycondensation at $180{ }^{\circ} \mathrm{C}$ followed by thermal decomposition at 220 ${ }^{\circ} \mathrm{C}$ under reduced pressure. Crude glycolide (G) monomer was synthesized from glycolic acid $(99 \%$, Acros Organics) by the same procedure. The reaction temperatures for the direct polymerization and the thermal decomposition stages were $220^{\circ} \mathrm{C}$ and 320 ${ }^{\circ} \mathrm{C}$, respectively, for preparing the crude $\mathrm{G}$ monomer. Crude lactide and glycolide monomers were purified by re-crystallization in ethyl acetate four times. The purified monomers were dried under vacuum at $55^{\circ} \mathrm{C}$ for $48 \mathrm{~h}$ before use in the polymerization. 1-dodecanol (98\%, Fluka) containing a one-hydroxyl end group was purified by distillation under reduced pressure before use. Stannous octoate [ $\mathrm{Sn}(\mathrm{Oct})_{2}$, $95 \%$, Sigma], indomethacin $(99.95 \%$, Sigma), and Tween 80 (Acros Organics) were used without further purification. All reagents used were analytical grade.

\section{Synthesis of copolymers}

Poly(D,L-lactide) (PDLL) and poly(D,Llactide-co-glycolide) (PDLLG) copolymers were synthesized by ring-opening polymerization of the DLL/LL/G mixtures in bulk at $165^{\circ} \mathrm{C}$ for $2.5 \mathrm{~h}$ under a nitrogen atmosphere. $\mathrm{Sn}(\mathrm{Oct})_{2}$ was used as a catalyst at $0.01 \mathrm{~mol} \%$ and 1 -dodecanol was used as an initiator. Copolymers with a theoretical numberaverage molecular weight $\left(M_{n, \text { theoretical }}\right)$ of $50,000 \mathrm{~g} /$ $\mathrm{mol}$ were prepared. The 1-dodecanol concentrations of 0.28 and $0.27 \mathrm{~mol} \%$ were used to synthesize the PDLL and the PDLLG, respectively. The crude copolymers were purified by dissolving in chloroform before precipitating in cool $n$-hexane. They were then dried to a constant weight in a vacuum at $50^{\circ} \mathrm{C}$ for $48 \mathrm{~h}$.

\section{Characterization of copolymers}

The specific optical rotation of the PDLL copolymers was determined in chloroform at a concentration of $1 \mathrm{~g} / \mathrm{dL}$ at $25^{\circ} \mathrm{C}$ with a Bellingham and Stanley Polarimeter ADP220 at a wavelength of $589 \mathrm{~nm}$. The specific optical rotation of the PDLL 
was used to calculate the $D$ and $L$ enantiomer contents. ${ }^{7-8}$

The chemical compositions of the PDLLG copolymers were measured by ${ }^{1} \mathrm{H}-\mathrm{NMR}$ spectrometry using a Bruker Advance DPX 300 ${ }^{1} \mathrm{H}-\mathrm{NMR}$ spectrometer at $25^{\circ} \mathrm{C} . \mathrm{CDCl}_{3}$ was used as the solvent, and tetramethysilane was used as the internal standard.

The number-average molecular weight $\left(\mathrm{M}_{\mathrm{n}}\right)$ and molecular weight distribution (MWD) of the copolymers were determined by Gel Permeation Chromatography (GPC) with a Waters e2695 separations module equipped with PLgel $10 \mathrm{~mm}$ mixed $\mathrm{B} 2$ columns operating at $40^{\circ} \mathrm{C}$ and employing a refractive index $(\mathrm{RI})$ detector. Tetrahydrofuran was used as the solvent at a flow rate of $1.0 \mathrm{~mL} / \mathrm{min}$.

The thermal transition properties of the copolymers were determined with a Perkin-Elmer Pyris Diamond differential scanning calorimeter (DSC) under a nitrogen flow. For DSC, copolymers of $5-10 \mathrm{mg}$ in weight were heated at $10^{\circ} \mathrm{C} / \mathrm{min}$ over a temperature range of 0 to $200 \mathrm{C}$ ( $1^{\text {st }}$ heating scan) to observe their melting temperature $\left(\mathrm{T}_{\mathrm{m}}\right)$. Then the samples were quenched to $0^{\circ} \mathrm{C}$ according to the

Table 1: Molecular weight characteristics of copolymers from GPC curves

\begin{tabular}{lcc}
\hline $\begin{array}{l}\text { Comonomer ratio } \\
\text { (by mole) }\end{array}$ & $\begin{array}{c}\mathbf{M}_{\mathbf{n}} \\
(\mathbf{g} / \mathbf{m o l})\end{array}$ & MWD \\
\hline DLL/LL ratio & & \\
$100 / 0$ & 53,200 & 2.2 \\
$90 / 10$ & 49,600 & 2.7 \\
$80 / 20$ & 53,600 & 2.5 \\
$70 / 30$ & 52,100 & 2.4 \\
$60 / 40$ & 47,100 & 1.6 \\
$50 / 50$ & 49,200 & 1.8 \\
DLL/LL/G ratio & & \\
$75 / 0 / 25$ & 51,500 & 1.6 \\
$67.5 / 7.5 / 25$ & 56,400 & 1.5 \\
$60 / 15 / 25$ & 53,600 & 2 \\
$52.5 / 22.5 / 25$ & 47,600 & 1.7 \\
$45 / 30 / 25$ & 48,700 & 1.9 \\
$37.5 / 37.5 / 25$ & 51,200 & 1.7 \\
\hline
\end{tabular}

DSC instrument's own default cooling mode before heating from 0 to $200^{\circ} \mathrm{C}$ ( $2^{\text {nd }}$ heating scan) to observe their glass transition temperature $\left(T_{g}\right)$. The $T_{m}$ was measured as the peak value of the endothermal phenomena in the DSC curve. The $\mathrm{T}_{\mathrm{g}}$ was taken as the midpoint or half of the heat capacity increment, associated with the glass-to-rubber transition.

\section{Preparation of drug-loaded copolymer microparticles}

The copolymer microparticles entrapping the indomethacin model drug were prepared by the oil-in-water emulsion solvent evaporation method. Dichloromethane was used as an organic solvent. 90 $\mathrm{mg}$ of copolymer and $10 \mathrm{mg}$ of indomethacin were dissolved in $2.5 \mathrm{~mL}$ of dichloromethane (oil phase). The oil phase was slowly added-drop wise into 400 $\mathrm{mL}$ of a $2 \% \mathrm{w} / \mathrm{v}$ Tween80 solution in distilled water (water phase) under magnetic stirring. The organic solvents were evaporated in a fume hood for $6 \mathrm{~h}$. The drug-loaded microparticles suspended in the water phase were obtained. The resulting microparticles were collected by centrifugation before freezedrying.

\section{Characterization of drug-loaded copolymer microparticles}

The morphology of the microparticles was observed by scanning electron microscopy (SEM, JEOL JSM-6460LV). The microparticles were sputter-coated with gold to enhance the surface conductivity before scanning. The average size of the microparticles was determined from several SEM images by counting a minimum of 100 particle diameters using the smile view software (version 1.02).

The drug loading of the microparticles was measured by UV-vis spectrophotometry (Lamda 25, Perkin Elmer). For this purpose, the drugloaded microparticles were completely dissolved in dichlorometane before analysis with a UV-vis spectrophotometer at $\lambda_{\max }=319 \mathrm{~nm} .{ }^{9}$ The amount of indomethacin was calculated by comparison with a standard equation of indomethacin solution in dichloromethane. The standard equation and $\mathrm{R}^{2}$ were $y=0.0181 x+0.0158$ and 0.9996 , respectively. 
The theoretical drug loading content ( $\left.\mathrm{DLC}_{\text {theoretical }}\right)$, actual drug loading content ( $\left.\mathrm{DLC}_{\text {actual }}\right)$, and drug loading efficiency (DLE) were calculated form Equations (1) - (3), respectively. The DLC ${ }_{\text {actual }}$ was an average value from three measurements.

$$
\operatorname{DLC}_{\text {terecetical }}(\%)=\frac{\text { Weight of feed drug }}{\text { Weights of feed drug and PDLLA }} \times 100
$$

$\operatorname{DLC}_{\text {actual }}(\%)=\frac{\text { Weight of entrapped drug in microparti cles }}{\text { Weight of microparti cles }} \times 100$

$\operatorname{DLE}(\%)=\frac{\mathrm{DLC}_{\text {actual }}}{\mathrm{DLC}_{\text {theoretica }}} \times 100$

where the weight of the entrapped drug in the microparticles was measured by dissolving the drug-loaded microparticles in dichloromethane before analysis with UV-vis spectrophotometry at $\lambda_{\max }=319 \mathrm{~nm}$.

\section{In vitro drug release tests}

An in vitro drug release test with the microparticles was performed. About $10 \mathrm{mg}$ of drugloaded microparticles were placed in a pretreated dialysis bag before being incubated in a flask containing $200 \mathrm{~mL}$ of $0.02 \mathrm{M}$ phosphate buffer saline (PBS, pH 7.4). The flasks were kept in a shaker incubator at $37^{\circ} \mathrm{C}$ and $100 \mathrm{rpm}$ for $48 \mathrm{~h}$. At each desired time, some supernatant was withdrawn and replaced with an equal volume of fresh PBS medium. The release concentration of the indomethacin in the supernatant was determined by a UV-vis spectrophotometer at $\lambda_{\max }=319 \mathrm{~nm}^{9}$.

The amount of indomethacin model drug was calculated by comparison with a standard equation of indomethacin solution in PBS. The standard equation and $R^{2}$ were $y=0.02 x+0.0047$ and 0.9994 , respectively. The cumulative release

Table 2: Enantiomer ratios and chemical compositions of copolymers

\begin{tabular}{|c|c|c|c|c|}
\hline $\begin{array}{l}\text { Comonomer ratio } \\
\text { (by mole) }\end{array}$ & $\begin{array}{c}\text { Feed } \\
\mathrm{X}_{\mathrm{D}} / \mathrm{X}_{\mathrm{L}} \text { ratio } \\
\text { (by mole) }^{\mathrm{a}}\end{array}$ & $\begin{array}{c}\text { Final } \\
X_{D} / X_{L} \text { ratio } \\
\text { (by mole) }^{b}\end{array}$ & $\begin{array}{c}\text { Feed } \\
\text { DLL-LL/G ratio } \\
\text { (by mole) }^{c}\end{array}$ & $\begin{array}{c}\text { Final } \\
\text { DLL-LL/G ratio } \\
\text { (by mole) }^{d}\end{array}$ \\
\hline \multicolumn{5}{|l|}{ DLL/LL ratio } \\
\hline $100 / 0$ & $50 / 50$ & $50 / 50$ & - & - \\
\hline $90 / 10$ & $45 / 55$ & $45 / 55$ & - & - \\
\hline $80 / 20$ & $40 / 60$ & $41 / 59$ & - & - \\
\hline $70 / 30$ & $35 / 65$ & $37 / 63$ & - & - \\
\hline $60 / 40$ & $30 / 70$ & $32 / 68$ & - & - \\
\hline $50 / 50$ & $25 / 75$ & $27 / 73$ & - & - \\
\hline \multicolumn{5}{|l|}{ DLL/LL/G ratio } \\
\hline $75 / 0 / 25$ & - & - & $75 / 25$ & $75 / 25$ \\
\hline $67.5 / 7.5 / 25$ & - & - & $75 / 25$ & $76 / 24$ \\
\hline $60 / 15 / 25$ & - & - & $75 / 25$ & $78 / 22$ \\
\hline $52.5 / 22.5 / 25$ & - & - & $75 / 25$ & $76 / 24$ \\
\hline $45 / 30 / 25$ & - & - & $75 / 25$ & $72 / 28$ \\
\hline $37.5 / 37.5 / 25$ & - & - & $75 / 25$ & $72 / 28$ \\
\hline
\end{tabular}

a Calculated from feed DLL/LL ratio based on 50/50 D-/L-form ratio and 5/95 D-/L-form ratio for D,L-lactic acid and L-lactic acid, respectively.

${ }^{b}$ Determined from polarimetry method.

c Calculated from feed DLL/LL/G ratio.

${ }^{\mathrm{d}}$ Calculated from ${ }^{1} \mathrm{H}-\mathrm{NMR}$ spectra. 
percentage of indomethacin (\% drug release) was calculated based on the ratio of the drug release at each time and the initial drug content in the microparticles. The drug release profiles were plotted

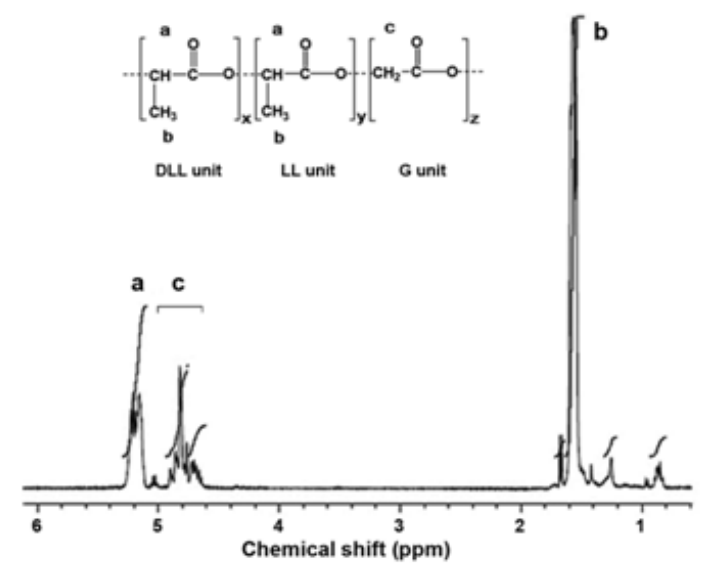

Fig. 1: ${ }^{1} \mathrm{H}-\mathrm{NMR}$ spectrum of PDLLG with feed DLL/LL/G mole ratio of 67.5/7.5/25 in $\mathrm{CDCl}_{3}$ (peak assignments as shown)
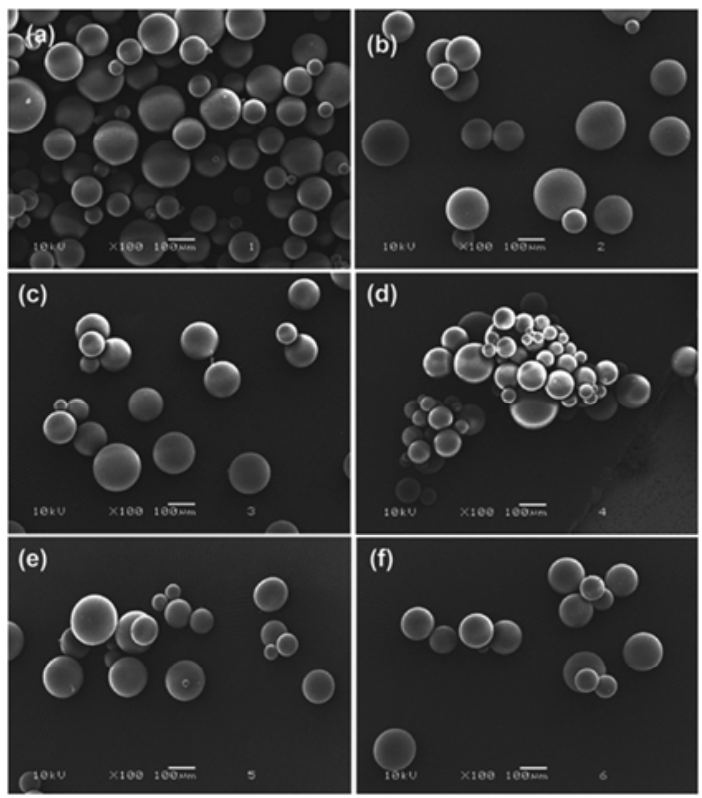

Fig. 2: SEM images of drug-loaded PDLL microparticles before drug release test prepared from PDLL with feed DLL/LL ratios of (a) 100/0, (b) 90/10, (c) 80/20, (d) 70/30, (e) 60/40, and (d) $50 / 50$ by mole (all scale bars $=100 \mathrm{~mm}$ ) between \%drug release and release time. The in vitro drug release tests were performed in triplicate.

\section{RESULTS AND DISCUSSION}

\section{Characterization of copolymers}

The yields of all the copolymers obtained from the precipitation method were higher than $85 \%$. Table 1 reports the number-average molecular weight $\left(M_{n}\right)$ and molecular weight distributions (MWD) of the copolymers obtained from the GPC curves. All the GPC curves were unimodal. The $M_{n}$ and MWD values were in the ranges of $47,100-56,400 \mathrm{~g} / \mathrm{mol}$ and 1.5-2.7, respectively. The $M_{n}$ values obtained from the GPC were close to the values of the theoretical $M_{n}(50,000 \mathrm{~g} / \mathrm{mol})$. The $M_{n}$ of the copolymers was then controlled by the 1-dodecanol concentration.

The PDLL copolymers with different DLL/ $L L$ ratios were polymerized from the mixtures of $D L L$ and LL monomers. The feed DLL/LL ratios were $100 / 0,90 / 10,80 / 20,70 / 30,60 / 40$, and $50 / 50$ by mole, which corresponded to the feed $X_{D} / X_{L}$ ratios
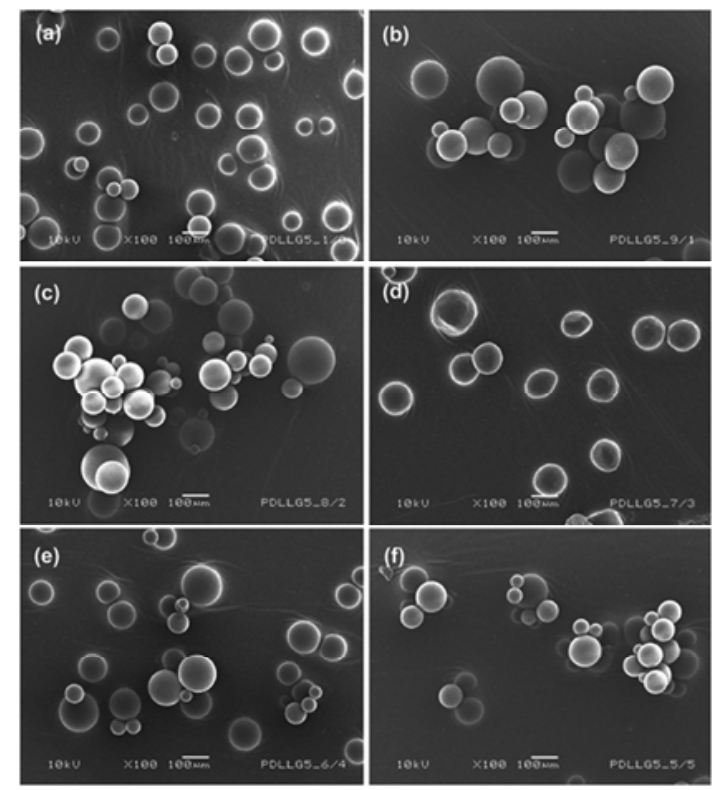

Fig. 3: SEM images of drug-loaded PDLLG microparticles before drug release test prepared from PDLLG with feed DLL/LL/G ratios of (a) 75/0/25, (b) 67.5/7.5/25, (c) 60/15/25, (d) 52.5/22.5/25, (e) $45 / 30 / 25$, and (f) $37.5 / 37.5 / 25$ by mole (all scale bars $=100 \mathrm{~mm}$ ) 
of $50 / 50,45 / 55,40 / 60,35 / 65,30 / 70$, and $25 / 75$ by mole, respectively, as summarized in Table 2 . The final $X_{D} / X_{L}$ ratios of the $P D L L$ copolymers determined from the polarimetry are also reported in Table 2. They were very close to the values of the feed $X_{D} / X_{L}$ ratios. The results suggest that the PDLL copolymers with different $X_{D} / X_{L}$ ratios can be synthesized from the DLL/LL mixtures.

The PDLLG copolymers with different DLL/ $L L / G$ ratios were synthesized from the mixtures of DLL, LL, and G momomers. The DLL/LL/G ratios were 70/0/25, 62.5/7.5/25, 60/15/25, 52.5/22.5/25,
$45 / 30 / 25$, and $37.5 / 37.5 / 25$ by mole, as reported in Table 2. The feed DLL-LL/G ratio was constant at $75 / 25$ by mole. The final DLL-LL/G ratios were determined from the ${ }^{1} \mathrm{H}$-NMR spectrum, an example of which is shown in Figure 1 for the 62.5/7.5/25 DLL/ LL/G copolymer including peak assignments. It can be seen that the half DLL and LL units exhibited the same peaks as the methine protons $(-\mathrm{CH}$; peak a) and methyl protons $\left(-\mathrm{CH}_{3}\right.$; peak b) at 5.2 and 1.5 ppm, respectively. ${ }^{10}$ The DLL/LL ratios of the PDLL copolymers could not be determined from the ${ }^{1} \mathrm{H}$ NMR spectra. The half $G$ units showed peaks of methylene protons $\left(-\mathrm{CH}_{2}\right.$; peak $\left.\mathrm{c}\right)$ in the range 4.6-

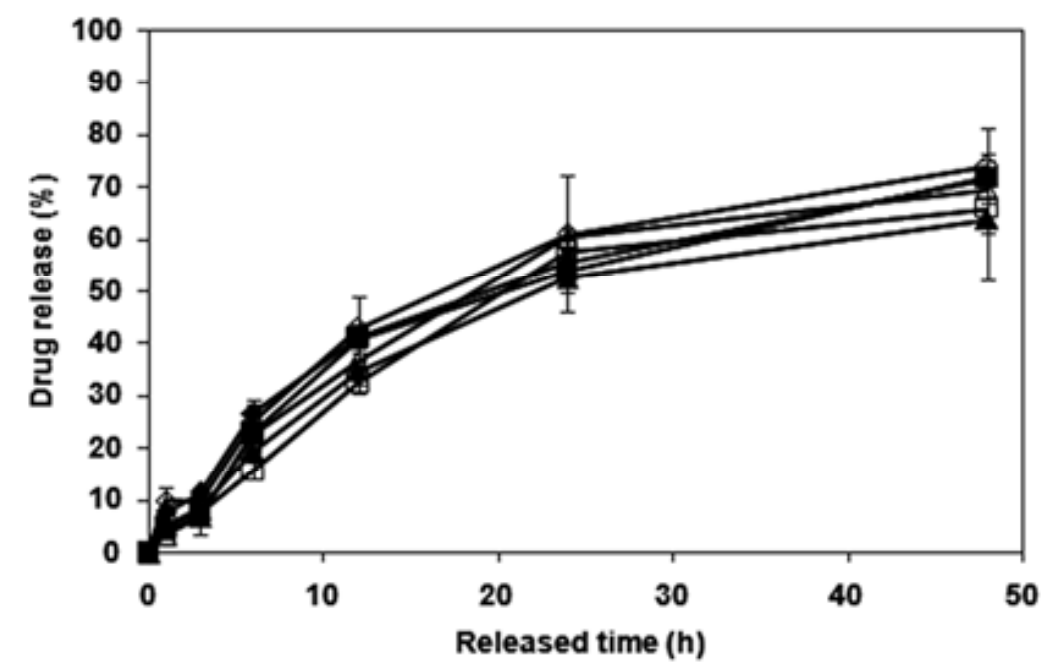

Fig. 4: In vitro drug release profiles of PDLL microparticles prepared from PDLL with feed DLL/LL ratios of $(\diamond)$ 100/0, $(\Delta)$ 90/10, ( ) 80/20, (•) 70/30, ( $\triangle$ ) 60/40, and ( $\square$ ) 50/50 by mole

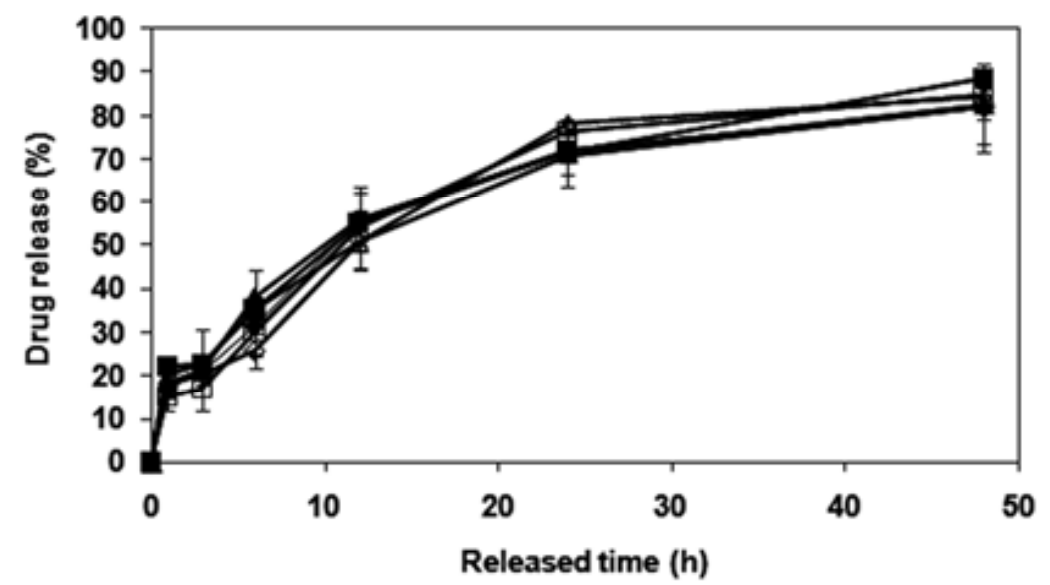

Fig. 5: In vitro drug release profiles of PDLLG microparticles prepared from PDLLG with feed

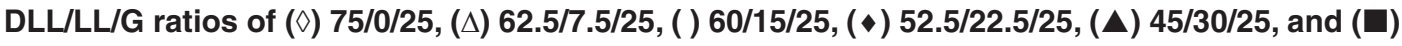
$37.5 / 37.5 / 25$ by mole 
$5.0 \mathrm{ppm} .{ }^{11}$ The final DLL-LL/G ratios of the PDLLG copolymers calculated from the integral peak areas of the peaks $\mathrm{a}$ and $\mathrm{c}$ are also reported in Table 2. They were nearly the same values as with the feed DLL-LL/G ratio ( $75 / 25$ by mole).

From the $1^{\text {st }}$ heating scan DSC curves, the melting peaks of all the PDLL and the PDLLG copolymers were not found (DSC thermograms not shown). This suggests that the copolymers with the DLL/LL and the DLL/LL/G ratios in the ranges of this study were completely amorphous. It is well known that amorphous polyesters are more appropriate for use in drug delivery. The entrapped drug could be uniformly distributed into the amorphous matrix better than the semi-crystalline matrix. This enhances the consistent drug release. The glass transition temperatures $\left(T_{g}\right)$ of the copolymers obtained from the $2^{\text {nd }}$ heating scan DSC curves were similar and in the ranges of $53-54^{\circ} \mathrm{C}$ and $48-49^{\circ} \mathrm{C}$ for
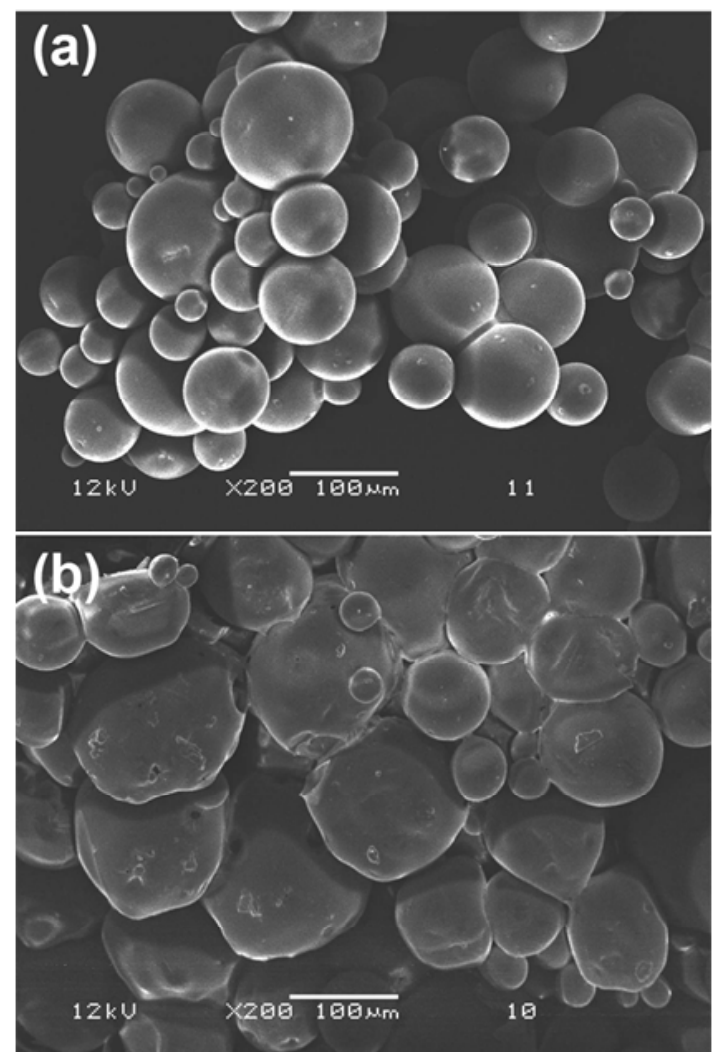

Fig. 6: SEM images of drug-loaded copolymer microparticles after $48 \mathrm{~h}$ release time prepared from (a) 100/0 DLL/LL, and (b) 75/0/25 DLL/LL/G copolymers (all scale bars $=100 \mathrm{~mm}$ ) the PDLL and the PDLLG copolymers, respectively. The results indicate that the DLL/LL and DLL/LL/G ratios do not affect the $T_{g}$ values of the copolymers. The $T_{g}$ values of the PDLLG copolymers were slightly lower than the PDLL copolymers because the $T_{g}$ of the homopolymers of polyglycolide $\left(37^{\circ} \mathrm{C}\right)$ was lower than the polylactide $\left(55^{\circ} \mathrm{C}\right)$.

\section{Characterization of copolymer microparticles}

The drug-loaded copolymer microparticles of PDLL and PDLLG were prepared by the oil-inwater emulsion solvent evaporation method. The yields of the microparticles, based on the weights of the feed copolymer and drug, were in the range of $82.4-93.7 \%$. The morphology of the drug-loaded microparticles was observed from the SEM images, as shown in Figures 2 and 3 for the PDLL and the PDLLG, respectively. They were spherical in shape with a smooth surface. The average particle sizes determined from several SEM images are summarized in Table 3. They were in the range of $89-113 \mathrm{~mm}$. The morphology results suggest that the monomer ratio of the copolymers did not significantly affect their particle morphology and size.

The theoretical drug loading content $\left(\mathrm{DLC}_{\text {theoretical }}\right)$ of all the copolymer microparticles calculated from Equation (1) was $10 \mathrm{wt} \%$. The actual drug loading content ( $\mathrm{DLC}_{\text {actual }}$ ) and the drug loading efficiency (DLE), as summarized in Table 3 , were in the ranges of $5.39-6.60 \%$ and $53.9-66.0 \%$, respectively. Both DLC ${ }_{\text {actual }}$ and DLE did not change significantly with the DLL/LL and the DLL/LL/G ratio.

\section{In vitro drug release}

The in vitro drug release test was performed in PBS at $37^{\circ} \mathrm{C}$ for $48 \mathrm{~h}$. The release profiles of indomethacin are illustrated in Figure 4 for the PDLL microparticles. All PDLL microparticles with different DLL/LL ratios showed similar sustained drug release patterns. An initial burst release of the drug near the particle surfaces was detected within the first $12 \mathrm{~h}$ followed by a slower drug release. The initial burst release of the PDLL microparticles was in the range of $30-40 \%$. The drug release was in the range of $60-70 \%$ at $48 \mathrm{~h}$ of release time.

Figure 5 shows the drug release profiles of the PDLLG microparticles. All PDLLG microparticles 
Table 3: Characteristics of drug-loaded copolymer microparticles

\begin{tabular}{|c|c|c|c|}
\hline $\begin{array}{l}\text { Comonomer ratio } \\
\text { (by mole) }\end{array}$ & $\begin{array}{l}\text { Average particle size } \\
\qquad(\mathrm{mm})^{\mathrm{a}}\end{array}$ & $\begin{array}{l}\text { DLC }_{\text {actual }} \\
(\%)^{b}\end{array}$ & $\begin{array}{l}\text { DLE } \\
(\%)^{c}\end{array}$ \\
\hline \multicolumn{4}{|l|}{ DLL/LL ratio } \\
\hline $100 / 0$ & $104 \pm 34$ & $5.93 \pm 0.04$ & 59.3 \\
\hline $90 / 10$ & $109 \pm 30$ & $5.50 \pm 0.10$ & 55 \\
\hline $80 / 20$ & $111 \pm 32$ & $6.08 \pm 0.03$ & 60.8 \\
\hline $70 / 30$ & $94 \pm 23$ & $5.75 \pm 0.34$ & 57.5 \\
\hline $60 / 40$ & $101 \pm 37$ & $5.76 \pm 0.14$ & 57.6 \\
\hline $50 / 50$ & $113 \pm 27$ & $5.39 \pm 0.18$ & 53.9 \\
\hline \multicolumn{4}{|l|}{ DLL/LL/G ratio } \\
\hline $75 / 0 / 25$ & $89 \pm 16$ & $5.63 \pm 0.02$ & 56.3 \\
\hline $67.5 / 7.5 / 25$ & $102 \pm 21$ & $6.21 \pm 0.17$ & 62.1 \\
\hline $60 / 15 / 25$ & $93 \pm 23$ & $6.09 \pm 0.33$ & 60.9 \\
\hline $52.5 / 22.5 / 25$ & $103 \pm 13$ & $6.27 \pm 0.18$ & 62.7 \\
\hline $45 / 30 / 25$ & $92 \pm 29$ & $6.12 \pm 0.14$ & 61.2 \\
\hline $37.5 / 37.5 / 25$ & $92 \pm 22$ & $6.60 \pm 0.08$ & 66 \\
\hline $\begin{array}{l}\text { a Determined from s } \\
\text { b Calculated from Ec } \\
{ }^{c} \text { Calculated from Ec }\end{array}$ & $\begin{array}{l}\text { veral SEM images } \\
\text { ation (2) } \\
\text { ation (3) }\end{array}$ & & \\
\hline
\end{tabular}

with different DLL/LL/G ratios exhibited similar sustained drug release profiles; an initial burst release effect within the first $12 \mathrm{~h}$ followed by slower drug release. However, the ranges of the initial burst release and drug release at $48 \mathrm{~h}$ of the PDLLG microparticles were $50-60 \%$ and $80-90 \%$, respectively. It can be concluded that the DLL/LL and DLL/LL/G ratios did not significantly influence the drug release behaviors of the PDLL and the PDLLG microparticles, respectively. However the PDLLG microparticles exhibited a faster drug release than those of the PDLL microparticles. The drug release results suggest that the PDLL and PDLLG copolymers with different monomer mole ratios showed potential for use as controlled release drug delivery systems. The concentrations of the drug in the plasma could be maintained at the therapeutic level for longer periods of time. Therefore, the frequency of drug administration could be reduced.

The predominant drug release mechanism of the PDLL microparticles was proposed to be the drug diffusion process. This was supported by the SEM image of the PDLL microparticles in Figure 6(a). The PDLL microparticles after $48 \mathrm{~h}$ release were still spherical in shape and had a smooth surface. Meanwhile the PDLLG microparticles had surface erosion, as shown in Figure 6(b). Thus the drug release mechanisms of the PDLLG microparticles within $48 \mathrm{~h}$ may include both drug diffusion and surface erosion. ${ }^{10}$ This may be due to the higher hydrophilicity and the lower $\mathrm{T}_{\mathrm{g}}$ of the PDLLG microparticle matrices that gave easier water penetration into the particle matrices. Therefore, higher water penetration induces faster surface erosion.

\section{CONCLUSIONS}

PDLL and PDLLG copolymers with different DLL/LL and DLL/LL/G mole ratios, respectively, were successfully synthesized via ring-opening polymerization of the mixtures of DLL, LL, and $G$ monomers. They were completely amorphous. The drug-loaded copolymer microparticles of the PDLL and the PDLLG were prepared by the oil-inwater emulsion solvent evaporation method. These amorphous microparticles could be used to entrap a poorly-water soluble model drug, indomethacin with a $89-113 \mathrm{~mm}$ size and a $53.9-66.0 \%$ loading efficiency for drug delivery. All the copolymer microparticles were spherical in shape and had a 
smooth surface. The copolymerization of the LL units was as high as 50 and $37.5 \mathrm{~mol} \%$ for the PDLL and the 75/25 PDLLG copolymers, respectively, did not induced PLLA crystallization, and did not affect the drug release profiles significantly. The PDLLG microparticles showed faster drug release than the PDLL microparticles.

In conclusion, the results presented here show that the amorphous PDLL and PDLLG copolymers synthesized in this work have the potential to be developed further as drug delivery systems. The amorphous PDLL and PDLLG copolymers with a high $L$ enantiomer content could provide a viable lower-cost alternative to the commercial PDLL and PDLLG.

\section{ACKNOWLEDGEMENTS}

This work was supported by the Division of Research Facilitation and Dissemination, Mahasarakham University (2015). The Center of Excellence for Innovation in Chemistry $(\mathrm{PERCH}-$ $\mathrm{CIC}$ ), Office of the Higher Education Commission, Ministry of Education, Thailand is also acknowledged. The authors are very grateful to Dr. Jolyon Dodgson, Department of Biology, Faculty of Science, Mahasarakham University for his improvement of the English language in this manuscript.

\section{REFERENCES}

1. Langer, R., New methods of drug delivery. Science, 1990; 249:1527-1533.

2. Champion, J.A.; Katare, Y.K.; Mitragotri, S., Particle shape: a new design parameter for micro- and nanoscale drug delivery carriers. J. Controlled Release, 2007; 121:3-9.

3. Anderson, J.M.; Shive, M.S., Biodegradation and biocompatibility of PLA and PLGA microspheres. Adv. Drug Deliv. Rev., 1997; 28:5-24.

4. Freiberg, S.; Zhu, X.X., Polymer microspheres for controlled drug release. Int. J. Pharm., 2004; 282:1-18.

5. Liang, R.; Li, X.; Shi, Y.; Wang, A.; Sun, K.; Liu, W.; Li, Y., Effect of water on exenatide acylation in poly(lactide-co-glycolide) microspheres. Int. J. Pharm., 2013; 454:344-353.

6. Acharya, A.P.; Lewis, J.S.; Keselowsky, B.G., Combination co-encapsulation of hydrophobic molecules in poly(lactide-coglycolide) microparticles. Biomaterials, 2013; 34:3422-3430.

7. Tsuji, H.; Wada, T.; Sakamoto, Y.; Sugiura, Y., Stereocomplex crystallization and spherulite growth behavior of poly(L-lactide)-b-poly(Dlactide) stereodiblock copolymers. Polymer, 2010; 51:4973-4947.

8. Kumar, S.; Bhatnagar, N.; Ghosh, A.K., Effect of enantiomeric monomeric unit ratio on thermal and mechanical properties of poly(lactide). Polymer Bulletin, 2016; 73:2087-2104.

9. Srisuwan, Y.; Baimark, Y., Biodegradable poly(D,L-lactide)/lipid blend microparticles prepared by oil-in-water emulsion method for controlled release drug delivery. Orient. J. Chem., 2014; 30:63-69.

10. Y Baimark Y.; Srisa-ard, M., Preparation of Drug-Loaded Microspheres of Linear and Star-Shaped Poly(D,L-lactide)s and Their Drug Release Behaviors, J. Appl. Polym. Sci., 2012; 124:3871-3878.

11. Baimark, Y.; Srisa-ard, M.; Puntumchai, A. Synthesis of poly(D,L-lactic acid-co-glycolic acid-co-e-caprolactone) terpolyesters by direct polycondensation, Current Res. Chem., 2010; 2:10-17. 